\title{
Línea de base de Responsabilidad Social Empresarial en Florencia, Caquetá. Una oportunidad de mejora*
}

\section{Baseline of Corporate Social Responsibility (CSR) in Florencia, Caquetá. An opportunity for improvement}

\section{Linha de base da Responsabilidade Social Empresarial em Florência, Caquetá. Uma oportunidade para melhorar}

Yorcelis Yudith Cantillo Carrillo**

Compensar

Yina Bolena Toledo Polanía****

Secretaría de Salud Departamental del Caquetá

* Artículo de resultado de investigación. DOI: http://dx.doi.org/10.15332/s2145-1389.2017.0002.06

**Magíster en Calidad y Gestión Integral. Especialista en Hematología en el Laboratorio Clínico y Manejo del Banco de Sangre. Bacterióloga. Bacterióloga Hospital Barrios Unidos - Compensar. Correo electrónico: yorceliscantillo@yahoo.com.

*** Magíster en Calidad y Gestión Integral. Especialista en Gerencia y Auditoría de la Calidad en Salud. Administradora de Empresas. Contratista en la Secretaría de Salud Departamental del Caquetá. Correo electrónico; yina.toledo@yahoo.es 


\section{RESUMEN}

La investigación aquí tratada corresponde a un estudio cualitativo aplicado a nivel empresarial en la capital del Caquetá para establecer una línea base de Responsabilidad Social Empresarial (RSE) a la luz de la norma ISO 26000, Guía sobre responsabilidad social. Esta guía, aplicable a todo tipo de organización, orienta sobre cómo integrar el comportamiento socialmente responsable, dentro de las estrategias, sistemas y procesos institucionales, a través del desarrollo de principios y materias fundamentales de RSE. Fueron estos los principales aspectos a evaluar en una muestra de 37 organizaciones del sector productivo de Florencia, mediante encuestas realizadas a gerentes y directivos, así como por medio de la observación de evidencias de gestión. El estudio, que siguió la metodología planteada por Medianero Burga (2011) para la realización de una línea de base, identificó prácticas de RSE emprendidas por las organizaciones y estableció el nivel de desarrollo de dichas acciones. Los resultados obtenidos dejan ver que, a pesar de un evidente interés por parte de los directivos de las empresas, el desarrollo de la RSE en Florencia es incipiente, pues se limita a acciones que nacen de la obligatoriedad de la ley o, en el mejor de los casos, hacen parte de las mismas estrategias de las empresas.

Palabras clave: línea base, responsabilidad social, principios y temas de la ISO 26000.

\section{ABSTRACT}

The research here treated corresponds to a study applied in the capital of the department of Caquetá, to establish a baseline of Corporate Social Responsibility (CSR) in the light of the standard ISO 26000 Guide on social responsibility. This norm, applicable to all type of organization, guides on how to integrate the socially responsible behavior, within the strategies, systems and institutional processes, through the development of principles and fundamental subjects of Social Responsibility, so these the main aspects to be evaluated in a sample of 37 organizations of the productive sector of Florencia, through surveys made to managers and directors and documentary review of their management reports. The study, which followed the methodology proposed by Medianero Burga (2011) for the realization of a baseline, identified CSR practices undertaken by organizations and establish the level of development of such actions. The results obtained show that, despite an interest shown by managers of the companies, the development of the CSR in the city of Florencia is very incipient because it is limited to actions that arise from the obligation of the law or at best they are part of the same strategies of the companies.

Keywords: baseline, social responsibility, principles and topics of ISO 26000.

\section{RESUMO}

A pesquisa aqui tratada corresponde a um estudo qualitativo aplicado a nível empresarial na capital de Caquetá para estabelecer uma base de Responsabilidade Social Empresarial (RSE) guiada pela padrão ISO 26000 Guia sobre responsabilidade social. Esta guia, é aplicável a todo tipo de organização, orienta sobre como integrar o comportamento socialmente responsável, dentro das estratégias, sistemas e processos institucionais, através do desenvolvimento de princípios e questões fundamentais da RSE. Estes foram os principais aspectos a serem avaliados em uma apresentação de 37 organizações do setor produtivo de Florência, através de enquetes realizadas a gerentes e dirigentes, bem como através da observação de 
evidências de gestão. $\mathrm{O}$ estudo, que seguiu a metodologia criada por Medianero Burga (2011) para a realização de uma linha de base, identificou as práticas da RSE empreendidas pelas organizações e estabeleceu o nível de desenvolvimento dessas ações. Os resultados obtidos revelam que, apesar do interesse demonstrado pelos gestores das empresas, o desenvolvimento da RSE em Florência é incipiente, pois se limita às ações que nascem da obrigatoriedade da lei, ou, no melhor dos casos, fazem parte das mesmas estratégias da empresas.

Palavras-chave: linha de base, responsabilidade social, ISO 26000.

\section{INTRODUCCIÓN}

La Responsabilidad Social Empresarial (RSE), también conocida como Responsabilidad Social Corporativa (RSC), es una estrategia tan importante hoy en día para las organizaciones que su implementación constituye el aval de la sostenibilidad, crecimiento, expansión y posicionamiento de las empresas a largo plazo.

No obstante, a pesar de la acelerada evolución del tema a nivel mundial, la falta de conocimientos sobre el sentido de la responsabilidad social, el carácter voluntario del alcance contenido en la norma ISO 26000 y la falta de un liderazgo estatal para promover acciones responsables socialmente, hace que muchas zonas del mundo permanezcan rezagadas con pobres prácticas asistencialistas que distan de lo que plantea un verdadero desarrollo sostenible organizacional.

Para Chiavenato (2007), responsabilidad social significa el grado de obligaciones que una organización asume por medio de políticas, decisiones y acciones que proyectan y mejoren el bienestar de la sociedad. Por lo tanto, las empresas son factores determinantes en el propósito de lograr una sociedad desarrollada.

Ello pone de manifiesto la necesidad de avanzar cada vez más en la implementación de la responsabilidad social (RSE). Muchas investigaciones sobre el tema de RSE han sido realizadas en el mundo por diferentes organismos. Uno de los más recientes estudios es la Encuesta de responsabilidad social corporativa, de la firma KPMG International (2013), la cual proporciona una visión de las tendencias mundiales actuales para ayudar a las empresas a determinar sus propios enfoques a la hora de hacer informes, así como a evaluar y mejorar la calidad de sus reportes. También se encuentran informes realizados en Bolivia (Pimentel, 2008), Ecuador (Torresano, 2012) y Colombia (CCRE, 2006), que muestran que, aunque se ha logrado un considerable avance en esta materia, aún las empresas tienen un desempeño incipiente. Por su parte, la Asociación Nacional de Empresarios de Colombia -ANDI (2012) también sugiere realizar una línea de base y efectúa una encuesta de responsabilidad social hacia el año 2012 en la que se evidencia el avance de la RSE como modelo integral de gestión de las organizaciones.

En el Caquetá, un departamento marcado por innumerables factores demográficos, ambientales y administrativos (Alcaldía de Florencia, 2016), no se evidencia que las administraciones locales ni otros organismos hayan logrado implementar verdaderos programas que favorezcan el desarrollo integral de su comunidad, como lo sería la promoción de actividades socialmente responsables por parte de sus empresas. A pesar de constituir un ambiente favorable para el desarrollo de las mismas, la ausencia de estudios que permitan establecer desde dónde empezar a direccionar programas y planes estratégicos en este rumbo, deja mucho que desear, máxime cuando los programas de gobierno diseñados por las administraciones locales en el ámbito municipal y 
departamental, en los últimos cinco años, permiten ver líneas estratégicas exclusivas en pro del desarrollo sostenible de la ciudad.

Empero, no existen diagnósticos que reflejen la situación actual de la ciudad en materia de responsabilidad social ni documentos que sirvan de insumo para los planes de desarrollo de los administradores de turno; ni siquiera entidades como la Cámara de Comercio de Florencia o el Fondo Emprender del SENA, que propician la creación y el fortalecimiento de las empresas del departamento, han adelantado estudios al respecto, lo que deja en duda qué saben los ciudadanos y empresarios de la responsabilidad social o qué están aplicando en sus organizaciones.

La incertidumbre frente al tema sugiere la necesidad inminente de levantar información que sirva de base para, desde allí, sensibilizar y direccionar planes y programas que promuevan la RSE en el departamento, empezando por su capital, ya que un trabajo estructurado sobre el tema propiciará el camino hacia la construcción de estrategias para formar organizaciones competitivas y, con ello, el acercamiento hacia esquemas de desarrollo sostenible en la ciudad.

En tal sentido, se desarrolla una investigación aplicada cuyo objetivo principal consiste en establecer una línea base de RSE en la ciudad de Florencia (Caquetá) desde el enfoque de la norma ISO 26000 Guía de responsabilidad social, abordándola a partir de la realización de encuestas a gerentes y directivos de 37 empresas del sector público y privado de la ciudad. La encuesta indagó sobre la aplicación de principios y temas de la norma ISO 26000 (ISO, 2010), así como frente a prácticas desarrolladas por las empresas, para luego inferir si estas se limitaron al cumplimiento de la ley o si fueron más allá de ella, desarrollándose así los tres objetivos específicos de la investigación.

\section{METODOLOGÍA}

De acuerdo al método utilizado, se trata de uno del tipo cualitativo (Hernández, Fernández y Baptista, 2010), dado que recoge información respecto a los conocimientos, aplicación de principios y prácticas de RSE de las empresas de Florencia. Así mismo, el estudio también posee un componente cuantitativo, ya que requiere de la recolección de información a través de encuestas y observación que permiten medir y cuantificar las variables objeto de estudio.

Además, cuatro etapas dieron forma al estudio realizado. La primera etapa fue de planeación, en la que se definió el problema a tratar, el ámbito de aplicación, los objetivos, el marco referencial y la propuesta metodológica que delimitó las variables a evaluar, las técnicas, los instrumentos de estudio y el marco muestral. Se estableció que el área de estudio serían las organizaciones del sector productivo de la ciudad de Florencia, que el método de muestreo a utilizar consistiría en el muestreo probabilístico aleatorio -el cual arrojó una muestra de 37 empresas, 21 privadas y 16 públicas de diferentes sectores de la economía-, y que la encuesta y la observación serían las técnicas a utilizar para recolectar la información relacionada con el desarrollo de la responsabilidad social en las empresas de Florencia, objetivos del estudio.

La segunda etapa correspondió a la construcción del instrumento para la aplicación de la encuesta, el cual incluyó preguntas dirigidas a gerentes y directivos de las empresas seleccionadas, una validación por parte de tres expertos y una prueba piloto aplicada al $10 \%$ de la muestra, para finalmente constituir un cuestionario de 28 preguntas que evaluó la aplicación de principios y temas de la norma ISO 26000, así como los documentos bajo los cuales se legitiman esas acciones en las organizaciones de la capital del departamento del Caquetá.

La tercera etapa del estudio se desarrolló a partir del trabajo de campo o aplicación de la encuesta sobre 
fuentes primarias (organizaciones seleccionadas), información que fue complementada a través de la observación de evidencias de gestión de las organizaciones que afirmaron incluir el componente de RSE en sus plataformas estratégicas. Esta información fue de gran utilidad para recolectar las iniciativas y prácticas de responsabilidad social de las empresas de Florencia. Se puede decir que la encuesta contribuyó al desarrollo de los tres objetivos, pero el segundo fue concretado con observación de evidencias de gestión. Esta etapa culmina con la tabulación de los datos y con el análisis de la información recolectada.

Por último, con los resultados de la información recolectada, se construyó el documento final que ayudó a inferir las conclusiones anotadas en este documento y con las cuales se brindó la información a las autoridades locales y otros organismos como la Cámara de Comercio de Florencia, para reforzar la promoción de la RSE en sus agendas estratégicas.

\section{RESULTADOS Y DISCUSIÓN}

\author{
Aplicación de principios y temas fundamentales \\ de la norma ISO 26000.
}

La muestra de 37 empresas tanto públicas como privadas del sector productivo de la ciudad de Florencia (Caquetá), arrojó que estas aplican medianamente los principios de la norma ISO 26000. Se destaca una mayor aplicación del principio de legalidad, en el que el $93 \%$ de los encuestados responden "siempre" y "casi siempre" cumplir con el marco legal reglamentario en el ámbito nacional e internacional. En ese porcentaje tienen una participación muy parecida las empresas públicas y privadas.

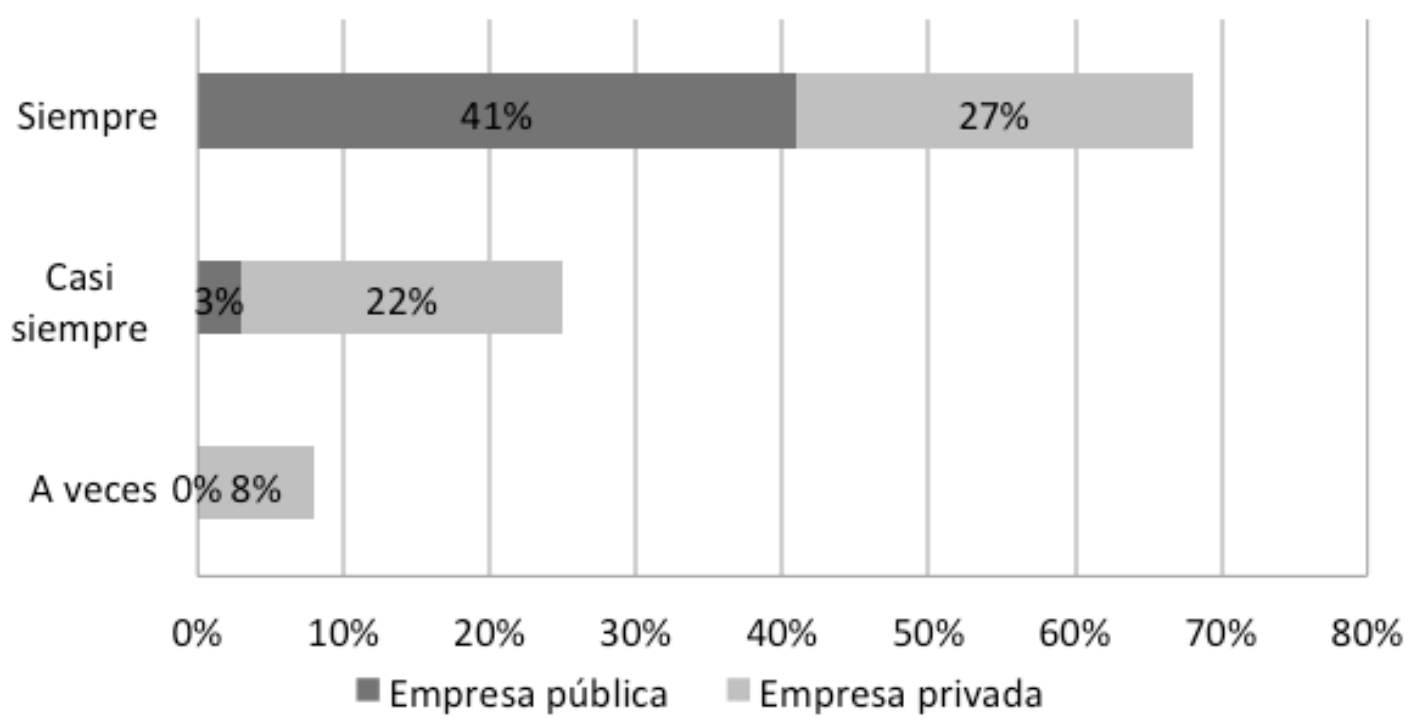

Fuente: las autoras.

Figura 1. Principio de legalidad.

El segundo principio mas aplicado es el "respeto por las partes de interés", en donde el $78 \%$ de las empresas, en una gran proporción pertenecientes al sector servicios, respondieron estar de acuerdo en la aplicación del mismo. 


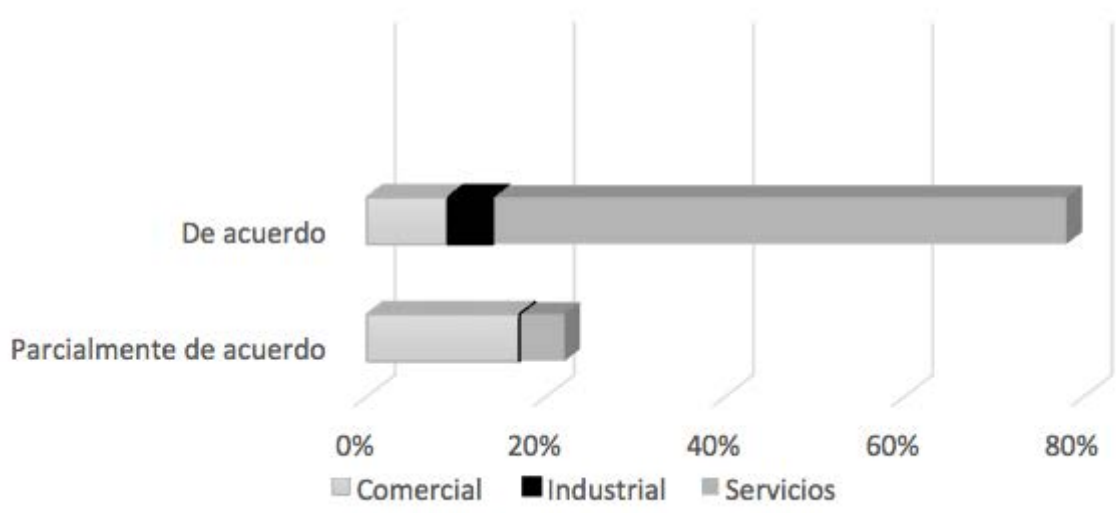

Figura 2. Principio respeto por las partes de interés.

Fuente: las autoras.

El principio menos desarrollado es el "respeto por los derechos humanos". El $62 \%$ de las empresas encuestadas desarrollan este tema a nivel sobresaliente, bueno. En estos temas las empresas públicas tienen mayor participación que las empresas privadas.

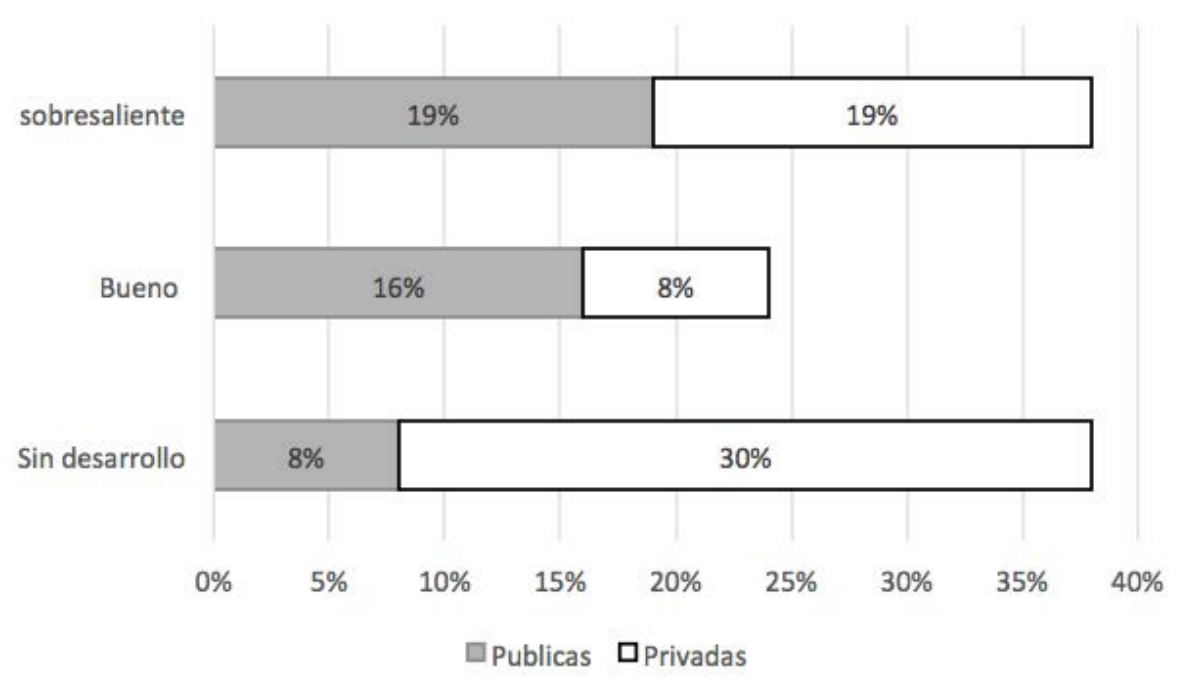

Figura 3. Respeto por los derechos humanos.

Fuente: las autoras.

Dentro de los temas más desarrollados por las organizaciones de Florencia se encuentran: las prácticas laborales, asuntos de consumidores y medio ambiente.
El tema menos desarrollado por las empresas de Florencia es el de prácticas justas de operación. 
Iniciativas y prácticas de las organizaciones de Florencia que reflejan actuaciones de RSE

En las organizaciones que incluyeron el componente de RSE en sus plataformas estratégicas, predomina el desarrollo de acciones frente al medio ambiente, las cuales en su mayoría corresponden a acciones voluntarias emprendidas por las organizaciones. También se detectan algunas prácticas elementales relacionadas con los consumidores, de las cuales la mayoría nacen de exigencias normativas, especialmente las establecidas para el sector salud.

Tabla 1. Prácticas de responsabilidad social en la ciudad de Florencia.

\begin{tabular}{|c|c|c|c|}
\hline TEMA & \multicolumn{3}{|c|}{ PRÁCTICAS DE RSE DE LAS EMPRESAS DE LA CIUDAD DE FLORENCIA } \\
\hline \multirow{2}{*}{ 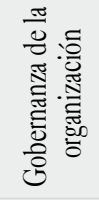 } & Uso de comités para despliegue de estrategias & \multirow{3}{*}{ 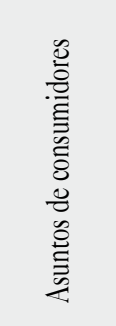 } & Ampliación de portafolio de productos o servicios \\
\hline & $\begin{array}{l}\text { Despliegue de metas y resultados a través de revista interna } \\
\text { y publicaciones en periódicos }\end{array}$ & & $\begin{array}{l}\text { Cambio de sistemas eléctricos tradicionales a } \\
\text { sistemas eléctricos seguros }\end{array}$ \\
\hline 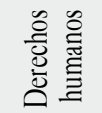 & $\begin{array}{l}\text { Aplicación de lineamientos nacionales frente a la protección } \\
\text { de derechos humanos }\end{array}$ & & $\begin{array}{l}\text { Implemantación de sistemas de gestión de la } \\
\text { calidad }\end{array}$ \\
\hline \multirow{9}{*}{ 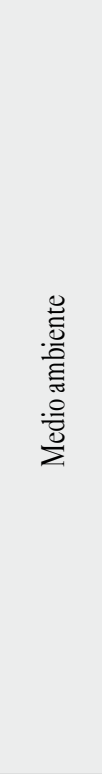 } & $\begin{array}{l}\text { Promoción de prácticas para el uso racional de los recursos } \\
\text { naturales (agua, energía, etc.) }\end{array}$ & \multirow{5}{*}{ 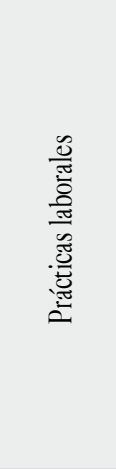 } & Implementación de programas de bienestar laboral \\
\hline & Implementación de programas de reciclaje & & $\begin{array}{l}\text { Campañas a empleados a favor de la promoción de } \\
\text { estilos de vida saludables }\end{array}$ \\
\hline & Manejo integral de residuos sólidos & & $\begin{array}{l}\text { Aplicación de normatividad en salud y seguridad } \\
\text { en el trabajo }\end{array}$ \\
\hline & Uso de bombillos ahorradores & & $\begin{array}{l}\text { Reconocimiento de desempeño a empleados y } \\
\text { áreas de la organización }\end{array}$ \\
\hline & $\begin{array}{l}\text { Instalación de sensores para racionamiento del servicio de } \\
\text { energía }\end{array}$ & & $\begin{array}{l}\text { Implementación de sistemas de seguridad y salud } \\
\text { ocupacional }\end{array}$ \\
\hline & Tratamiento de aguas residuales & \multirow{5}{*}{ 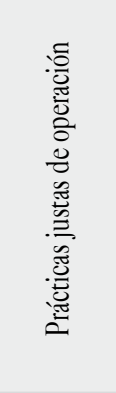 } & Adopción del código de ética y del buen gobierno \\
\hline & Implementación de buenas prácticas de manufactura & & Rendición de cuentas a la comunidad \\
\hline & Implementación de programas de producción más limpia & & $\begin{array}{l}\text { Utilización de sofware para compras justas y efi- } \\
\text { cientes }\end{array}$ \\
\hline & Implementación de sistemas de gestión ambiental & & Establecimiento de criterios para compras verdes \\
\hline \multirow{4}{*}{ 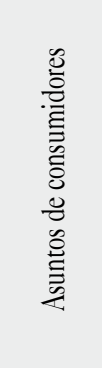 } & $\begin{array}{l}\text { Implementación de la oficina de atención al cliente } 0 \\
\text { usuario }\end{array}$ & & Fortalecimiento del Sistema de Control Interno \\
\hline & Educación en derechos y deberes de los usuarios & \multirow{3}{*}{ 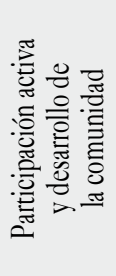 } & $\begin{array}{l}\text { Vinculación laboral de personal con discapacidad } \\
\text { física }\end{array}$ \\
\hline & $\begin{array}{l}\text { Capacitación de pacientes y familiares en temas de auto- } \\
\text { cuidado }\end{array}$ & & Generación de empleo \\
\hline & Implementación de la política de atención humanizada & & $\begin{array}{l}\text { Participación de la asociación de usuarios en pro- } \\
\text { yectos de desarrollo misional }\end{array}$ \\
\hline
\end{tabular}

Fuente: las autoras. 


\section{Nivel de desarrollo de la RSE en la ciudad de Florencia}

Los resultados obtenidos a través de las encuestas, nos permite inferir que el desempeño de las empresas de Florencia es aceptable en cuatro de los temas de la norma ISO 26000. Las mayores deficiencias se presentan en el tema de prácticas justas de operación, mientras que los mejores desempeños lo han alcanzado las empresas en el tema laboral.

Tabla 2. Nivel de desarrollo de la RSE en la ciudad de Florencia.

\begin{tabular}{lc}
\hline \multicolumn{1}{c}{ Temas } & $\begin{array}{c}\text { Proporción } \\
\text { de empresas } \\
(\%)\end{array}$ \\
\hline Gobernanza de la organización & 60 \\
\hline Derechos humanos & 62 \\
\hline Medio ambiente & 59 \\
\hline Prácticas justas de operación & 49 \\
\hline Prácticas laborales & 81 \\
\hline Contribución al desarrollo & 76 \\
económico y social & 70 \\
\hline Asunto de consumidores &
\end{tabular}

Nivel alcanzados por las
empresas
Sobresaliente: $81-100 \%$
Bueno: $71-80 \%$
Aceptable: $51-70 \%$
Deficiente: $0-50 \%$

Fuente: las autoras.

Un $50 \%$ de las iniciativas desarrolladas por las empresas corresponden a actividades que nacen de la obligatoriedad normativa y el otro $50 \%$ son de carácter voluntario, sin que se observe que estas últimas se traten de acciones que trasciendan las prácticas más sencillas y comúnmente usadas por las organizaciones en el ámbito de sus operaciones.

\section{CONCLUSIONES}

Los resultados de esta investigación demostraron que la RSE, a pesar de ser un tema de vital importancia, no solo a nivel estratégico para mejorar el nivel de competitividad de cualquier organización, sino para mejorar las condiciones socioeconómicas de la sociedad en general, no es manejada por la mayoría de las empresas del sector productivo de la ciudad de Florencia (Caquetá).

Se pudo analizar en la muestra estudiada que las empresas, directa 0 indirectamente, unas por iniciativa propia y otras por disposiciones legales, realizan acciones de Responsabilidad Social Empresarial como las relacionadas con la seguridad y salud en el trabajo, manejo de residuos y atención al cliente; sin embargo, consideran estas actividades como estrategias de competitividad y en muchos casos, obligaciones por cumplir. Al contrario, otras empresas justifican su responsabilidad social en el hecho de realizar acciones que no son de obligatorio cumplimiento, sin que ello signifique que se trate de actividades que trasciendan más allá de lo común (prácticas de reciclaje, calidad en los productos, uso de bombillos ahorradores).

La presente línea base realizada también deja ver que, a pesar de que los empresarios de Florencia tienen conocimientos de la RSE, son pocas las empresas que han incluido este componente en sus plataformas estratégicas, y aquellos que la tienen no consideran que la RSE esté declarada como verdadera política en sus organizaciones.

Entre las empresas públicas y las privadas no existe diferencia significativa en cuanto al alcance de las actividades de RSE, lo cual deja mucho que desear frente 
a la norma ISO 26000 Guía sobre Responsabilidad Social, que pretende que las organizaciones vayan más allá de lo legal. Para el caso de la muestra estudiada, cada sector implementa de acuerdo a la normatividad aplicable. Por ejemplo, lo relacionado con el tema ético, de transparencia y rendición de cuentas, es propio de las empresas públicas, por su obligatoriedad, al igual que lo concerniente a la participación y desarrollo de la comunidad, cuya responsabilidad le asiste por ser esta su población objeto. Las empresas privadas desarrollan más actividades enmarcadas en la normatividad aplicable en el tema ambiental y en aspectos relacionados con productos y servicios.

En las empresas de Florencia el principio más aplicado en las organizaciones es el de "respeto por las partes de interés". En una menor proporción el principio de "legalidad", y el menos aplicado es el "respeto por los derechos humanos".

Los temas más desarrollados por las organizaciones de Florencia son los relacionados con las "prácticas laborales", en menor proporción "asuntos de consumidores" y menor aún lo relacionado con "medio ambiente". El tema menos desarrollado por las empresas de Florencia es el de "prácticas justas de operación".

A pesar de que medianamente las organizaciones manejan principios y temas de RSE, la gran mayoría lo hacen basados en lo reglamentado por ley.

En el tema de gobernanza de la organización, las empresas despliegan sus planes y decisiones a través de comunicación interna. En el tema de derechos humanos las organizaciones se caracterizan por contar con los mecanismos básicos de ley; en materia laboral, por el estricto cumplimiento de las disposiciones legales; en el aspecto ambiental, por la realización de actividades de reciclaje y manejo adecuado de residuos; en lo referente a los usuarios, se caracterizan por contar con el servicio de atención al cliente y, finalmente, frente a la participación de la comunidad, por desarrollar proyectos que la involucren. Esto especialmente en las empresas del sector público.

Las empresas de la ciudad de Florencia están desarrollando actividades de responsabilidad social a nivel de cumplimiento de sus obligaciones legales y en el mejor de los casos, a nivel de los efectos que se derivan de su propia operación, debido a que en la mayoría de organizaciones la dimensión económica está por encima de las necesidades de la sociedad de la cual se hace parte.

La construcción de esta primera línea de base de RSE en la ciudad de Florencia deja ver que las empresas vienen realizando actividades enmarcadas dentro de los principios de RSE inmersos en las regulaciones existentes para cada negocio, pero aquellas que asienten las bases de un cambio cultural profundo y que aporten al desarrollo sostenible de la sociedad de la que hacen parte, tienen esta misión pendiente por realizar.

Ante los resultados obtenidos por la presente línea base de RSE desarrollada en la ciudad de Florencia, que ilustra la falta de un liderazgo de las administraciones locales para promover organizaciones socialmente responsables, la falta de conocimientos sobre el sentido de la responsabilidad social y el carácter voluntario del alcance contenido en la norma ISO 26000, nos permitimos exhortar a las empresas de la ciudad para liderar procesos internos de RSE, generando estrategias y adoptando políticas sobre la base del conocimiento, que conlleven a la generación de cadena de valor en las organizaciones de la región.

En el marco de las políticas públicas, es fundamental crear, mantener y coordinar canales de comunicación entre el gobierno, la academia, el sector productivo, las asociaciones, los partidos políticos y la sociedad civil, de manera que se genere un proceso en el cual los poderes y organizaciones públicas sean determinantes al momento de presentar un plan de desarrollo que un 
gobernante se comprometa a ejecutar, trayendo beneficios sociales y económicos a una comunidad.

Desde su papel de trazador de políticas, las administraciones públicas desarrollan una función dinamizadora, por tanto ejercer el liderazgo en procesos de responsabilidad social se convierte en una buena estrategia para conducir a la ciudad hacia esquemas de desarrollo sostenible.

Se sugiere establecer un sistema que estabilice y permita implementar una política pública de manera que se inicie todo un proceso de cultura y comprensión de la importancia de la responsabilidad social como factor generador de progreso y sostenibilidad de la capital y, con ella, del departamento del Caquetá.

\section{REFERENCIAS}

Alcaldía de Florencia. (2016). Plan de desarrollo 2016-2019. Recuperado de: http:// www.florencia-caqueta.gov.co/apc-aa-files/34376437646636323731373462646134/plan_ de_desarrollo_2016_2019.pdf

ANDI. (2012). Encuesta de responsabilidad social empresarial 2012. Asociación Nacional de Empresarios de Colombia. Recuperado de: https://masteradmon.files.wordpress.com/2013/10/informe-rse2012-octubre-2012_20130121_033950.pdf

CCRE. (2006). Linea de base sobre responsabilidad social empresarial en Colombia. Estudio 2006. Centro
Colombiano de Responsabilidad Social Empresarial. Bogotá, D. C., Colombia: CCRE e Ipsos-Napoleón Franco.

Chiavenato, I. (2007). Introducción a la teoría general de la administración. México D. F., México: McGraw-Hill.

Hernández Sampieri, R., Fernández Collado, C., y Baptista Lucio, P. (2010). Metodología de la investigación. México D. F., México: Mc Graw Hill.

ISO. (2010). Guía de responsabilidad social. Norma internacional ISO 26000. Organización Internacional de Normalización. Ginebra, Suiza: ISO.

KPMG International Cooperative. (2013). The KPMG Survey of Corporate Responsibility Reporting 2013. Recuperado de: www.kpmg.com/Global/en/ IssuesAndInsights/ArticlesPublications/corporate-responsibility/Documents/corporate-responsibility-reporting-survey-2013-exec-summary.pdf

Medianero Burga, D. (2011). Metodología de estudios de línea de base. Pensamiento Crítico, 15, 61-82.

Pimentel Rojas, E. (2008). Linea base de responsabilidad social empresarial. La Paz, Bolivia: Labor Consultores y Asociados.

Torresano, M. (2012). Estudio de responsabilidad social de empresas del Ecuador 2012. Recuperado de: www.redceres.org/wp-content/uploads/2014/02/ Estudio-de-Responsabilidad-Social-de-Empresas-en-Ecuador.pdf 